\title{
石墨烯及其复合材料在催化有机反应中的研究新进展
}

\author{
张丽高书涛刘伟华唐然肖商宁昭 \\ 王 春* 王志 \\ (河北农业大学理学院 保定 071001)
}

\begin{abstract}
摘要 石墨烯作为一种新型碳纳米材料, 由于其具有大的比表面积、良好的热稳定性和化学稳定性、易于进行化学修 饰等优点，基于石墨烯及其复合材料的催化体系得到了广泛的关注. 目前石墨烯及其复合材料已经成功地应用于催化 Suzuki-Miyaura, Heck, 硝基还原, 氨硼烷水解脱氢等一系列有机反应. 对近年来石墨烯及其复合材料催化的有机反应 的最新研究进展进行简要评述.
\end{abstract}

关键词 石墨烯; 有机合成; 催化; 综述

\section{Research Progress of Graphene and Its Composites in Organic Synthesis}

\author{
Zhang, Li Gao, Shutao Liu, Weihua Tang, Ranxiao Shang, Ningzhao \\ Wang, Chun* Wang, Zhi \\ (College of Science, Agricultural University of Hebei, Baoding 071001)
}

\begin{abstract}
As a novel carbon nanomaterial, graphene based composite has received much attention in catalysis due to its unique characterisics such as large surface area, good thermal and chemical stability, strong hydrophobicity, easy modification, etc. Graphene and its composites have been applied in the catalysis of different organic reactions, such as Suzuki-Miyaura, Heck, and reduction of nitroarenes. The research progress of graphene-based composites catalyst is briefly reviewed in the paper.
\end{abstract}

Keywords graphene; organic synthesis; catalysis; review

石墨烯 $(\mathrm{G})$, 一种新型 $\mathrm{sp}^{2}$ 杂化的二维碳纳米材料, 自 2004 年被英国 Manchester 大学的 Geim 等 ${ }^{[1]}$ 发现后, 就以其极好的物理化学性质在众多的科学技术领域产 生了极为深远的影响. 石墨烯本身具有大的比表面积 $\left(2630 \mathrm{~m}^{2} \cdot \mathrm{g}^{-1}\right)$ 、大的共轭体系、很强的疏水性、易于进 行功能化修饰、很好的耐酸、耐碱、耐热性能和化学稳 定性, 亦可与有机分子产生极强的 $\pi-\pi$ 相互作用. 石墨 烯可由天然石墨制备, 具有成本低廉、原料易得且容易 实现规模化制备的优点，石墨烯及其复合材料在电子、 信息、能源、材料、催化、吸附和生物医药等领域具有 广泛的应用前景. 石墨烯不仅可作为优异的催化剂载 体, 其本身也可用于催化系列有机反应. 目前石墨烯及 其复合材料已经成功地应用于催化 Suzuki-Miyaura,
Heck，硝基还原，氨硼烷水解脱氢等诸多有机反应. 本 文拟对近年来石墨烯及其复合材料催化的有机反应的 最新研究进展进行简要评述.

\section{1 石墨烯负载金属催化的有机反应}

\section{1 交叉偶联反应}

碳碳偶联反应是构建 $\mathrm{C}-\mathrm{C}$ 键最有效的方法，目前 已广泛应用于天然产物、医药、农药以及功能材料等的 合成. 2009 年, Scheuermann 等 ${ }^{[2]}$ 采用离子交换法制备 了氧化石墨烯负载钯催化剂(Pd-GO), 用于催化取代溴 苯与苯硼酸的 Suzuki 偶联反应(Eq. 1). 仅在 $0.25 \mathrm{~mol} \%$ 的 Pd-GO 催化下，产物的产率可高达 $81 \% \sim 100 \%$, 这

\footnotetext{
*E-mail: chunwang69@126.com

Received March 1, 2014; revised April 5, 2014; published online April 16, 2014

Project supported by the Natural Science Foundation of Hebei Province (No. B2011204051) and the Innovation Research Program of Department of Education of Hebei for Hebei Provincial Universities (No. LJRC009).

河北省自然科学基金(No. B2011204051)和河北省高等学校创新团队领军人才培育计划(No. LJRC009)资助项目.
} 
主要得益于氧化石墨烯载体的优良物化性质, 与传统催 化剂载体如活性炭、沸石、有机聚合物相比, 由于氧化 石墨烯具有大的比表面积、大的共轭体系, 以及含有大 量含氧基团(如羧基、羟基、环氧基等), 使得 $\mathrm{Pd}$ 纳米粒 子均匀地负载到氧化石墨烯上, 从而表现出较高的催化 活性. 但是, 此催化剂重复使用 4 次时, 产物产率仅为 $19 \%$, 这可能是由于采用离子交换法制备的催化剂过滤

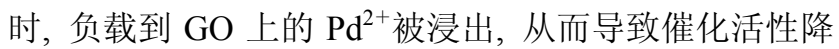
低.

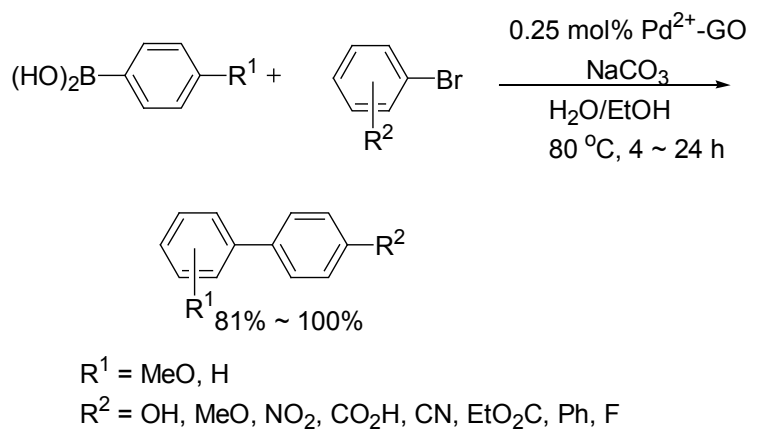

(1)

2010 年, Li 小组 ${ }^{[3]}$ 以十二烷基硫酸钠为表面活性剂, 首次将钯纳米粒子负载到石墨烯表面制得 Pd-G 催化剂, 用于催化碘苯与苯嗍酸在水相中的交叉偶联反应， 100 ${ }^{\circ} \mathrm{C}$ 下反应 $5 \mathrm{~min}$ 即可反应完全. 同时考察了钯纳米粒子 大小 $(4 \sim 15 \mathrm{~nm})$ 对催化活性的影响, 结果表明直径小的 钯粒子的催化活性和选择性都高于直径大的钯粒子, 这 主要归因于直径小的钯粒子可以形成更多的催化位点, 除此之外, Pd 纳米粒子和石墨烯的较强相互作用, 也促 进了反应的进行. 另外, 采用水热法制备的 Pd-G 催化 剂重复使用 10 次, 反应产率仍达 $78.6 \%$. 同时, $\mathrm{Li}$ 小组 还讨论了 $\mathrm{Pd}^{2+}-\mathrm{G}$ 催化剂对溴苯、烯丙基碘与苯硼酸的 反应活性, 实验结果表明, 其催化活性低于碘苯与苯硼 酸的反应活性. 这可能是由于 $\mathrm{C}-\mathrm{Br}$ 和 $\mathrm{C}-\mathrm{I}$ 键的强度 不同, 另外, 卤素取代基的吸电子能力可能也会有一定 的影响.

$\mathrm{Li}$ 小组 ${ }^{[4]}$ 采用同样的方法首次制备出石墨烯负载的 金纳米催化剂(Au-G). 研究结果表明, Au-G 也有相对较 高的催化活性. 对于负载 $8 \mathrm{wt} \% \mathrm{Au}$ 的 $\mathrm{Au}-\mathrm{G}$ 催化剂, $\mathrm{Au}$ 纳米粒子直径为 $2 \sim 3 \mathrm{~nm}$ 时, 其催化碘苯与苯硼酸反应 的转化率和选择性分别为 $76.5 \%$ 和 $85.8 \%$; 相同反应条 件下负载 $21 \mathrm{wt} \% \mathrm{Au}$ 的 $\mathrm{Au}-\mathrm{G}$ 催化剂, $\mathrm{Au}$ 纳米粒子直径 为 $7.5 \mathrm{~nm}$ 时, 其催化同一反应的转化率和选择性分别为 $59.8 \%$ 和 $11.2 \%$, 这与 $\mathrm{Li}$ 小组对 $\mathrm{Pd}$ 纳米粒子的研究结果 一致. 另外, $\mathrm{Li}$ 小组也对烯丙基碘与苯硼酸反应进行了 研究, 其相应的转化率和选择性相对较低. Au-G 催化剂 的重复使用 5 次, 仅为初始催化活性的 $73.4 \%$.

2011 年, Siamaki 等 ${ }^{[5]}$ 首次采用微波辅助法合成
Pd-G 催化剂. 与传统的加热方法相比, 微波辅助法加热 均匀, 从而使高浓度的钯纳米颗粒更好地分散在石墨烯 的表面. Pd-G 催化剂对 Suzuki 交叉偶联反应表现出较高 的催化活性、选择性和重复使用性(Eq. 2). 在微波加热 条件下, 卤代芳烃的转化率可达 $108000 \mathrm{~h}^{-1}$, 而且 Pd-G 催化剂对 Heck 反应也有较高的催化活性(Eq. 3), 例如, 以澳苯为反应原料时, 其产物产率高达 $97 \%$.
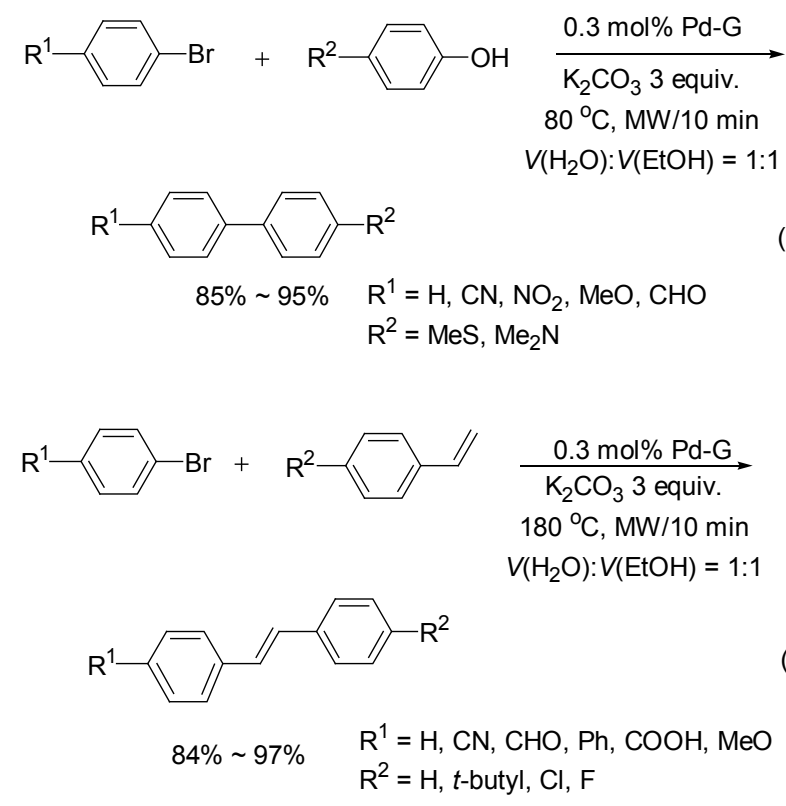

2012 年, $\mathrm{He}$ 等 ${ }^{[6]}$ 首次直接利用 $\mathrm{AuCl}_{4}^{-}$和 $\mathrm{GO}$ 制得 $\mathrm{Au}-\mathrm{GO}$. 以溴苯与苯嗍酸的偶联反应为例, 仅在 0.3 mol\%的催化剂作用下, 联苯产率可达 97\%. 除此之外, 催化剂 Au-GO 重复使用 6 次, 其催化活性仍为原始催化 活性的 91\%. 这可能是由于氧化石墨烯表面含有大量的 含氧官能团，碱性条件下带负电荷，这样带正电荷的金 属阳离子很容易进入层间与氧化石墨烯形成配位键，因 而在催化剂使用过程中, $\mathrm{Au}$ 不易被浸出损失.

2013 年, Shendage 等 ${ }^{[7]}$ 首次采用电化学方法制备催 化剂 Pd-RGO, 并用于催化 Suzuki 交叉偶联反应. 以 $\mathrm{K}_{2} \mathrm{CO}_{3}$ 为碱, 乙醇为反应溶剂在回流条件下, 产物的最 高产率可达 95\%. 在此条件下, 以 Pd-RGO 催化剂对一 系列卤代苯进行研究, 结果表明芳基碘代物反应完全需 较短的反应时间, 芳基溴代物则需相对较长的反应时 间，而且吸电子基芳基卤代物比给电子基芳基卤代物的 产物产率高(Eq. 4). 另外, 催化剂 Pd-RGO 的稳定性也 较好, 重复使用 6 次, 其催化活性仍为原始催化活性的 $86 \%$.

同年, Kim 等 ${ }^{[8]}$ 首次以抗坏血酸为还原剂, 采用一 步还原法成功合成贵金属 $(\mathrm{Pd}, \mathrm{Pt}, \mathrm{Au}, \mathrm{Ag})$ /石墨烯催化 剂，从而避免了使用有毒有害的还原剂如 $\mathrm{NaBH}_{4}$ 、水合 


$$
\begin{aligned}
& \mathrm{R}^{1}=\mathrm{CH}_{3}, \mathrm{OCH}_{3}, \mathrm{NO}_{2}, \mathrm{COCH}_{3}, \mathrm{OH}, \mathrm{H} \\
& \mathrm{R}^{2}=\mathrm{CH}_{3}, \mathrm{OCH}_{3}, \mathrm{H} \\
& X=\mathrm{I}, \mathrm{Br}
\end{aligned}
$$$$
\underset{\substack{\mathrm{K}_{2} \mathrm{CO}_{3} \text {, ethanol } \\ 1 \sim 2.5 \mathrm{~h} \text {, reflux }}}{\stackrel{\mathrm{Pd}-\mathrm{RGO}}{\longrightarrow}}
$$

肼. 然而, 采用抗坏血酸为还原剂需较长的还原时间, 另外, 抗坏血酸的量也会影响石墨烯表面金属纳米粒子 的大小和形状, 甚至可能导致纳米颗粒的团聚, 降低催 化剂的催化活性. 在催化 Suzuki 交叉偶联反应中, 需在 $110{ }^{\circ} \mathrm{C}$ 下反应 $48 \mathrm{~h}$, 且产率相对较低.

2012 年, $\mathrm{Hu}$ 研究小组 ${ }^{[9]}$ 首次把 $\mathrm{Fe}_{3} \mathrm{O}_{4}$ 和 $\mathrm{Pd}$ 纳米粒 子负载到磺化后的石墨烯(s-G)表面, 以 Suzuki-Miyaura 交叉偶联反应为例, 产物产率在 $45 \mathrm{~min}$ 内即可达到 $88 \%$ \% $\%$. 研究还表明, $\mathrm{Pd} / \mathrm{Fe}_{3} \mathrm{O}_{4} / \mathrm{s}-\mathrm{G}$ 不仅可高效催化 吸电子基取代的芳基溴化物, 还可催化给电子基取代的 芳基溴化物(Eq. 5). 催化剂 $\mathrm{Pd} / \mathrm{Fe}_{3} \mathrm{O}_{4} / \mathrm{s}-\mathrm{G}$ 在水溶液中分 散性好, 易于经磁场分离. 该催化剂还具有较高的重复 使用性能，当重复使用 8 次时，仅损失了初始活性的 $16 \%$.

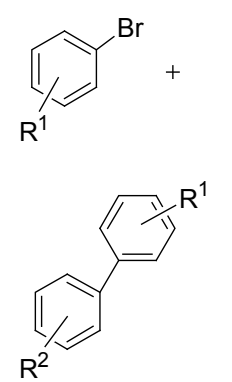

$88 \% \sim 95 \%$

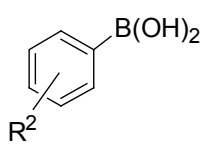

$\frac{0.3 \mathrm{~mol} \% \mathrm{Pd} / \mathrm{Fe}_{3} \mathrm{O}_{4} / \mathrm{s}-\mathrm{G}}{\longrightarrow}$
$\begin{gathered}80^{\circ} \mathrm{C}, 45 \mathrm{~min} \\ V\left(\mathrm{H}_{2} \mathrm{O}\right): V(\mathrm{EtOH})=1: 1\end{gathered}$

2013 年, Shendage 等 ${ }^{[10]}$ 首次采用电化学法将 Pd 纳 米粒子负载到经全氟磺酸修饰后的石墨烯上 $(\mathrm{Pd} / \mathrm{Nf}-\mathrm{G})$, 用于催化 Suzuki 交叉偶联反应(Eq. 6), 利用电化学方法 合成催化剂不仅对环境友好且不会对产物的结构性质 产生不良影响. 该小组对实验的反应条件如: 溶剂、温 度等进行了系列优化. 在优化条件下对不同的卤代芳烃 进行了研究, 催化剂 $\mathrm{Pd} / \mathrm{Nf}-\mathrm{G}$ 都表现出极好的催化活性, 产物产率在 $88 \% \sim 96 \%$ 之间.

2013 年, 我们小组 ${ }^{[11]}$ 首次将钯纳米颗粒负载到用 多胺修饰的氧化石墨烯上 $\left(\mathrm{GO}-\mathrm{NH}_{2}-\mathrm{Pd}^{2+}\right)$. 以 $\mathrm{GO}-\mathrm{NH}_{2}-$ $\mathrm{Pd}^{2+}$ 为催化剂, Suzuki-Miyaura 交叉偶联反应产物产率 可达 71\% 95\% (Eq. 7). 此外, 催化剂 GO-NH $\mathrm{NH}_{2}-\mathrm{Pd}^{2+}$ 重 复使用 10 次催化活性无明显降低.

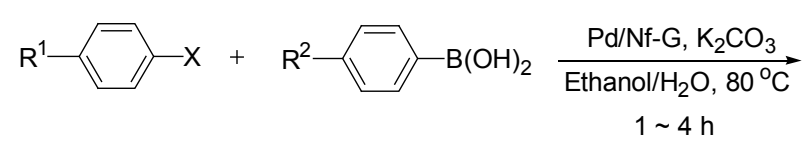

$$
\begin{aligned}
& \mathrm{R}^{1}=\mathrm{CH}_{3}, \mathrm{OCH}_{3}, \mathrm{NO}_{2}, \mathrm{COCH}_{3}, \mathrm{OH}, \mathrm{H} \\
& \mathrm{R}^{2}=\mathrm{CH}_{3}, \mathrm{OCH}_{3}, \mathrm{H} \\
& \mathrm{X}=\mathrm{I}, \mathrm{Br}
\end{aligned}
$$

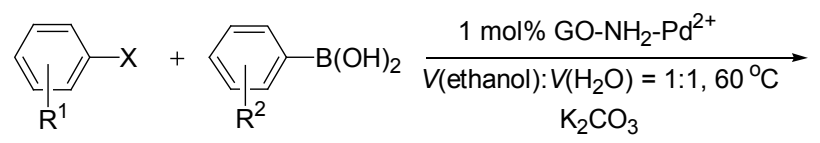

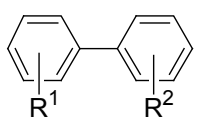

$71 \% \sim 95 \%$

$$
\begin{aligned}
& \mathrm{R}^{1}=\mathrm{H}, \mathrm{MeO}, \mathrm{EtOO}, \mathrm{NO}_{2}, \mathrm{CH}_{3} \\
& \mathrm{R}^{2}=p-\mathrm{H}, m-\mathrm{NO}_{2}, p-\mathrm{F} \\
& \mathrm{X}=\mathrm{I}, \mathrm{Br}
\end{aligned}
$$

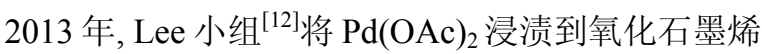
溶液中制备 $\mathrm{Pd}^{2+} / \mathrm{GO}$, 然后在 $100{ }^{\circ} \mathrm{C}$ 下用 $\mathrm{H}_{2}$ 还原 $2 \mathrm{~h}$, 得到的 $\mathrm{Pd} / \mathrm{G}$ 催化剂用于催化 Sonogashira 交叉偶联反 应. 与 $\mathrm{Pd} / \mathrm{GO}$ 相比, $\mathrm{Pd} / \mathrm{G}$ 催化剂具有更好的催化性能, 以碘苯和苯乙炔的反应为例, $\mathrm{Pd} / \mathrm{GO}$ 为催化剂产物的产 率为 $54 \%$, 而使用 $\mathrm{Pd} / \mathrm{G}$ 时则为 $89 \%$, 这主要归因于高度 分散的 Pd 纳米粒子使参与催化反应的活化位点增加, 故反应得以快速进行. 此外, 他们还探讨了 Pd 纳米粒子 的大小与催化活性之间的关系, 即粒径越小, 其催化活 性越高. $\mathrm{Pd} / \mathrm{G}$ 催化剂的重复使用率稍低, 重复使用 5 次 时, 仅为原始催化活性的 $46 \%, \mathrm{Pd} / \mathrm{G}$ 催化剂失活可能主 要归因于干燥过程中 Pd 纳米粒子的团聚.

\section{2 嗍胺烷的水解脱氢反应}

水解硼胺烷制备氢气是储氢工业最有前途的发展 方向之一. 由于硼胺烷相对分子质量低 $\left(30.9 \mathrm{~g} \cdot \mathrm{mol}^{-1}\right)$, 且含氢量高 $(19.6 \mathrm{wt} \%)$, 因此通过简单的化学催化嗍胺 烷溶液产氢成为人们的研究热点 ${ }^{[13]}$.

2012 年, Kilic 等 ${ }^{[14]}$ 首次采用液相浸渍法将 Pd 纳米 粒子负载到还原氧化石墨烯(RGO)上, 在催化水解硼胺 烷的反应中, Pd/RGO 催化剂的转化频率为 $26.3 \mathrm{~min}^{-1}$, 且在 $46 \mathrm{~h}$ 内可连续转化 11600 次(Eq. 8), 这是目前在所 有催化嗍胺烷水解反应中转化频率最高的催化剂, 甚至 比含 $\mathrm{Pt}$ 催化剂的转化频率还高. 此外, Pd/RGO 重复使 用 10 次, 其催化活性仍达原始活性的 $95 \%$. 如此高的重 复使用率可能是由于氧化石墨烯在还原过程中, 由于产 生 $\mathrm{CO}$ 和 $\mathrm{CO}_{2}$ 而生成许多空洞和拓扑缺陷, 缺陷越多, 电子复合机会越大，而金属离子的负载，正好使其与石 墨烯之间形成一种牢固的配位键，使负载的金属离子不 会因为后处理过程中过滤和离心而浸出. 
$\mathrm{H}_{3} \mathrm{NBH}_{3}+2 \mathrm{H}_{2} \mathrm{O} \quad \frac{(2.1 \text { wt } \% \mathrm{Pd}) \mathrm{Pd} / \mathrm{RGO}}{\text { r.t. }}\left(\mathrm{H}_{4} \mathrm{~N}\right) \mathrm{BO}_{2}+3 \mathrm{H}_{2} \quad$ (8)

同年, Metin 小组 ${ }^{[15]}$ 也将 Pd 纳米粒子负载到经化学 修饰后的石墨烯(CDG)上, 用于催化水解嗍胺烷反应, 结果表明 $\mathrm{Pd}$ 粒子的存在是关键, 而与石墨烯的存在形 态无太大关系.

2013 年, Yang 小组 ${ }^{[16]}$ 在常温条件下以硼胺烷和硼 氢化钠的混合物为还原剂将 $\operatorname{Co}(0)$ 纳米粒子负载到石墨 烯上，与传统的还原剂嗍氢化钠相比，该法制备的 $\mathrm{Co} / \mathrm{G}$ 的催化活性更高. 催化动力学数据表明产氢转化频率 (TOF) 为 $13.8 \mathrm{~mol} \mathrm{H}_{2} \cdot \mathrm{min}^{-1}(\mathrm{~mol} \mathrm{Co})^{-1}$, 比所有非贵金属 催化剂的转化频率都要高, 甚至比某些含贵金属催化剂 的转化频率更高, 该反应的活化能为 $32.75 \mathrm{~kJ} \cdot \mathrm{mol}^{-1}$, 比 许多负载其他金属的催化剂的活化能都要低. 但是 $\mathrm{Co} / \mathrm{G}$ 催化剂的重复使用性低, 仅可重复使用 5 次.

2014 年, $\mathrm{CaO}$ 等 ${ }^{[17]}$ 采用一步法还原氯化钓(III)和六 水合氯化钴(II), 常温条件下首次合成 Ru@Co 核壳双金 属修饰的石墨烯催化剂 $(\mathrm{Ru} @ \mathrm{Co} / \mathrm{G}), \mathrm{Ru} @ \mathrm{Co} / \mathrm{G}$ 催化嗍胺 烷的产氢转化频率(TOF) 为 $344 \mathrm{~mol} \mathrm{H}_{2} \cdot \mathrm{min}^{-1}$ ( $\mathrm{mol}$ $\mathrm{Ru})^{-1}$, 远高于传统催化剂 $\mathrm{Ru} / \mathrm{G}, \mathrm{Co} / \mathrm{G}$, 双金属 $\mathrm{Ru}-\mathrm{Co} / \mathrm{G}$. 除此之外, $\mathrm{Ru} @ \mathrm{Co} / \mathrm{G}$ 重复使用 15 次时其催 化活性为原始催化活性的 51\%.

\section{3 硝基化合物的还原反应}

\subsection{1 硝基酚的还原}

有机胺是一种重要的合成中间体, 可以广泛地应用 于医药、农用化学品和聚合物等的制备. 2012 年, Li 小 组 ${ }^{[18]}$ 采用水热法通过自组装 $\mathrm{Au}$ /石墨烯材料首次合成 $\mathrm{Au}$ /石墨烯水凝胶柱，该水凝胶柱含有 $2.26 \mathrm{wt} \%$ 的 $\mathrm{Au}$, $6.94 \mathrm{wt} \%$ 的石墨烯和 $90.8 \mathrm{wt} \%$ 的水, 其对硝基酚的室温 还原反应表现出极好的催化活性, 其催化活性大约为海 绵状 $\mathrm{Au}$ 纳米粒子催化剂的 90 倍, $\mathrm{Au}$ 纳米粒子负载聚合 物催化剂的 14 倍. $\mathrm{Au}$ /石墨烯水凝胶柱的高催化活性可 能是由于: (1)水凝胶结构更有利于 $\mathrm{Au}$ 纳米颗粒分散. (2)石墨烯的高吸附能力使硝基酚附着催化剂表面，(3) 电子由石墨烯转移到 $\mathrm{Au}$ 纳米颗粒, 促进硝基酚的电子 吸收，进而加快了反应的进行.

同年, $\mathrm{Qu}$ 小组 ${ }^{[19]}$ 首次成功地将 $\mathrm{Ag}$ 纳米粒子和磁性 $\mathrm{Fe}_{3} \mathrm{O}_{4}$ 负载到石墨烯上, 用于催化硝基酚的室温还原反 应, $\mathrm{GO}-\mathrm{Ag}-\mathrm{Fe}_{3} \mathrm{O}_{4}$ 表现出较好的催化活性. 该磁性催化 剂易于分离, 重复使用第 10 次仍无明显催化活性的降 低.

除了负载单金属外, 2013 年, Gupta 小组 ${ }^{[20]}$ 将双金属 $\mathrm{Fe}-\mathrm{Au}$ 负载于氧化石墨烯上, 制备了可磁性分离的高效 催化剂 $\mathrm{Fe}-\mathrm{Au} / \mathrm{GO}$. 研究表明, $\mathrm{Fe}-\mathrm{Au} / \mathrm{G}$ 对硝基酚的还原
反应有极好的催化活性. 相同反应条件下，催化剂 $\mathrm{Fe}-\mathrm{Au} / \mathrm{G}$ 对 4-硝基酚的催化活性大于 2-硝基酚, 这可能 是由于较大的空间位阻作用影响了催化剂活化底物时 的取向所致.

\subsection{2 硝基苯的还原反应}

2012 年, $\mathrm{Nie}$ 等 ${ }^{[21]}$ 以乙二醇(EG)为还原剂制备了 $\mathrm{Pt} /$ 还原氧化石墨烯 $(\mathrm{Pt} / \mathrm{RGO})$, 用于催化芳硝基化合物的还 原加氢反应. 扫描电镜图谱显示, 与使用传统的还原剂 水合肼相比, $\mathrm{EG}$ 为还原剂制备的 $\mathrm{Pt} / \mathrm{RGO}$, 其 $\mathrm{Pt}$ 粒子表 现出更好的单分散性、且分布更均匀. 同时 Pt/RGO-EG 也表现出极好的催化活性(Eq. 9), 以硝基苯为例，0 ${ }^{\circ} \mathrm{C}$ 条件下以 $\mathrm{Pt} / \mathrm{RGO}-\mathrm{EG}$ 为催化剂, 苯胺的产量可达到 70.2 $\mathrm{mol}_{-\mathrm{AN}} /\left(\mathrm{mol}_{\mathrm{Pt}} \cdot \mathrm{min}\right)$, 是 $\mathrm{Pt} / \mathrm{CNTs}$ 的 12.5 倍, $\mathrm{Pt} / \mathrm{C}$ 的 19.5 倍. 研究还表明, 反应温度为 $20{ }^{\circ} \mathrm{C}$ 时, Pt/RGO-EG 的催 化活性将升至 $1138.3 \mathrm{~mol}_{-\mathrm{AN}} /\left(\mathrm{mol}_{-\mathrm{Pt}} \bullet \mathrm{min}\right)($ 表 1$)$, 然而, $\mathrm{Pt} / \mathrm{RGO}-\mathrm{EG}$ 的重复使用率稍低，重复使用 5 次后仅为初 始活性的 $72.2 \%$.

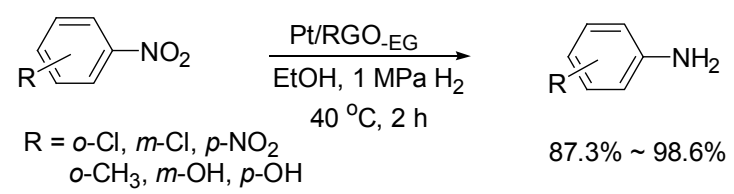

(9)

表 1 不同催化剂催化的硝基苯氢化反应 ${ }^{a}$

Table 1 The hydrogenation of nitroarenes to aniline with various catalysts

\begin{tabular}{|c|c|c|c|c|c|}
\hline Entry & Catalyst & $T /{ }^{\circ} \mathrm{C}$ & $t / \min$ & Yield/\% & $\begin{array}{c}\text { Activity [mol- }{ }_{-\mathrm{AN}} / \\
\left.\left(\mathrm{mol}-\mathrm{Pt}^{\bullet} \mathrm{min}\right)\right]\end{array}$ \\
\hline 1 & $\mathrm{RGO}_{-\mathrm{EG}}$ & 20 & 20 & 0 & - \\
\hline 2 & $\mathrm{Pt} / \mathrm{RGO}_{-\mathrm{HH}}$ & 0 & 180 & 0 & 0 \\
\hline 3 & $\mathrm{Pt} / \mathrm{RGO}_{\text {-EG }}$ & 0 & 180 & 94.3 & 70.2 \\
\hline 4 & $\mathrm{Pt} / \mathrm{MWCNT}_{-\mathrm{EG}}$ & 0 & 180 & 48.0 & 5.6 \\
\hline 5 & $\mathrm{Pt} / \mathrm{AC}-_{\mathrm{EG}}$ & 0 & 180 & 30.6 & 3.6 \\
\hline 6 & $\mathrm{Pt} / \mathrm{RGO}_{-\mathrm{EG}}$ & 20 & 20 & 99.1 & 663.8 \\
\hline 7 & $\mathrm{Pt} / \mathrm{MWCNT}_{-\mathrm{EG}}$ & 20 & 20 & 78.1 & 81.7 \\
\hline 8 & $\mathrm{Pt} / \mathrm{AC}_{-\mathrm{EG}}$ & 20 & 20 & 54.0 & 57.7 \\
\hline 9 & $\mathrm{Pt} / \mathrm{RGO}_{-\mathrm{EG}}{ }^{b}$ & 20 & 20 & 89.3 & 1138.3 \\
\hline
\end{tabular}

${ }^{a}$ Reaction conditions: $10.3 \mathrm{mmol}$ of nitrobenzene, $30 \mathrm{~mL}$ of ethanol, $20{ }^{\circ} \mathrm{C}$, 1.0 MPa, stir speed: $1000 \mathrm{r} / \mathrm{min} ;{ }^{b} 19.6 \mathrm{mmol}$ of nitrobenzene was added.

同年, $\mathrm{He}$ 等 ${ }^{[22]}$ 采用共沉淀法首次制得磁性氧化石 墨烯催化剂 $\left(\mathrm{Fe}_{3} \mathrm{O}_{4} / \mathrm{GO}\right)$, 用于催化芳硝基化合物的还原 加氢反应(Eq. 10). 研究结果表明 $\mathrm{Fe}_{3} \mathrm{O}_{4} / \mathrm{GO}$ 对一系列的 芳硝基化合物都有极好的催化作用，其转化频率(TOF) 高达 $3.63 \mathrm{~min}^{-1}$, 为商业 $\mathrm{Fe}_{3} \mathrm{O}_{4}$ 纳米催化剂的 45 倍, 且 $\mathrm{Fe}_{3} \mathrm{O}_{4} / \mathrm{GO}(3.1 \mathrm{wt} \%)$ 和水合肼 $(3.6 \mathrm{~mol} / \mathrm{L})$ 的用量均低于 已报道的 $\mathrm{Fe}_{3} \mathrm{O}_{4}(30.0 \mathrm{wt} \%)$ 和水合肼 $(5.0 \mathrm{~mol} / \mathrm{L})$ 的用量, 这可能是由于: (1) $\mathrm{Fe}_{3} \mathrm{O}_{4} \mathrm{NPs}$ 负载到氧化石墨烯上后, $\mathrm{Fe}_{3} \mathrm{O}_{4}$ 表面能降低, 更有利于氧化石墨烯上的一些含氧 官能团作为附着点对 $\mathrm{Fe}_{3} \mathrm{O}_{4}$ 纳米粒子进行吸附, 有利于 
$\mathrm{Fe}_{3} \mathrm{O}_{4}$ 的均匀分布. (2)由于氧化石墨烯具有大的比表面 积和吸附有机化合物能力, 这就增加了催化剂和反应试 剂之间的碰撞概率.

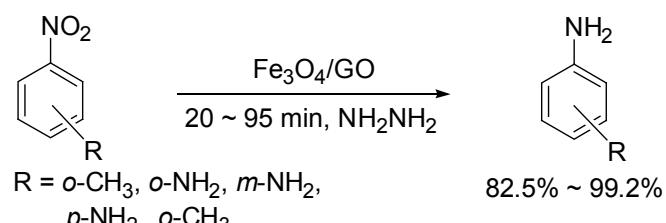

2013 年, Feng 小组 ${ }^{[23]}$ 同样采用共沉淀方法首次制 备了 $\mathrm{Fe}_{3} \mathrm{O}_{4} / \mathrm{G}$, 与 $\mathrm{Fe}_{3} \mathrm{O}_{4} / \mathrm{GO}$ 类似, $\mathrm{Fe}_{3} \mathrm{O}_{4} / \mathrm{G}$ 对硝基化合物 还原也有很好的催化作用，其产物产率为 75\% 92\% (Eq. 11). 重复使用 5 次, 无催化活性的损失.

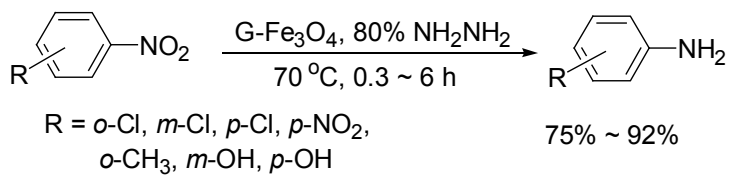

\section{4 石墨烯负载金属催化的其他反应}

\subsection{1 碳酸二甲酯的合成反应}

碳酸二甲酯(DMC)是一种无毒、环保性能优异、用 途广泛的化工原料. 2011 年, Bian 等 ${ }^{[24}$ 将双金属 $\mathrm{Cu}-\mathrm{Ni}$ 负载到石墨烯纳米薄片上(GNS)直接用于催化 DMC 的 合成, 实验结果表明, $\mathrm{Cu}-\mathrm{Ni} / \mathrm{GNS}$ 是一种具有较高催化 性能的催化剂, 相同反应条件下与一些有机金属催化剂 如金属的酚盐、无机催化剂如 $\mathrm{K}_{2} \mathrm{CO}_{3}, \mathrm{ZrO}_{2}, \mathrm{Cu}-\mathrm{Ni} / \mathrm{VSO}$ 和 $\mathrm{Cu}-(\mathrm{Ni}, \mathrm{V}, \mathrm{O}) / \mathrm{SiO}_{2}$ 等相比, 不仅其催化活性高, 而 且在 $\mathrm{DMC}$ 的脱水处理过程中, 干燥剂如 $\mathrm{CaCl}_{2}$ 等不会 对催化剂产生毒化作用. 另外, 其催化活性也比一些新 型催化剂高, 如比 $\mathrm{Cu}-\mathrm{Ni} / \mathrm{MWCNTs}$ 催化剂高 $4.3 \%$, 比 $\mathrm{Cu}-\mathrm{Ni} / \mathrm{TEG}$ 催化剂高 $4.97 \%$, 比 $\mathrm{Cu}-\mathrm{Ni}-\mathrm{V} / \mathrm{AC}$ 催化剂高 $7.39 \%$. 除此之外, Jun 小组还对反应温度、压强等进行 了优化.

\subsection{2 $\mathrm{CO}$ 的氧化反应}

$\mathrm{CO}$ 催化氧化反应是 $\mathrm{CO}$ 控释和减少排放的一类十 分重要的反应 ${ }^{[25]} .2012$ 年, $\mathrm{Li}$ 等 ${ }^{[26]}$ 采用浸渍法和氢气还 原法将 Pd 负载到石墨烯上制得 Pd 负载量分别为 $2 \%$, $6 \%$ 和 $10 \%$ 的 Pd-G 催化剂, 并用于催化 CO 的氧化反应, 其转化频率(TOF) $\left(100{ }^{\circ} \mathrm{C}, \times 10^{3} \mathrm{~s}^{-1}\right)$ 分别为 $2.63,3.89$ 和 4.88, 即 Pd 负载量越大, 催化剂催化活性越高. 升高 温度, 转化频率 TOF 值也会增大, 说明此反应为吸热反 应. 该反应的动力学数据表明其符合 LangmuirHinshelwood 原理. 另外, Pd-G 催化剂表现出较好的稳 定性, 这可能与石墨烯上的空穴有关, Pd 粒子会占据费 米能级附近的 $\mathrm{d}$ 轨道和石墨烯产生协同作用, 还可能和
石墨烯上存在的一些含氧基团有关.

同年, Zhang 等 ${ }^{[27]}$ 采用浸渍法分别制备了 Pt-Ni/G, $\mathrm{Pt} / \mathrm{G}$ 和 $\mathrm{Ni} / \mathrm{G}$ 三种催化剂, 结果表明, 三种催化剂催化活 性的高低顺序为: $\mathrm{Pt}-\mathrm{Ni} / \mathrm{G}>\mathrm{Pt} / \mathrm{G}>\mathrm{Ni} / \mathrm{G}$, 石墨烯本身无 催化活性.

\section{2 石墨烯催化的有机反应}

\section{1 硝基苯的还原反应}

负载金属的石墨烯可以催化硝基苯的还原反应，直 接以石墨烯为催化剂催化该反应也已有报道. 2011 年, 北京大学的 Gao 小组 ${ }^{[28]}$ 考察了还原氧化石墨烯室温条 件下催化的硝基苯还原反应, 仅使用少量催化剂, 苯胺 的产率即可达 $97.4 \%$. 而相同反应条件下，以石墨、膨 胀石墨、氧化石墨烯为催化剂, 苯胺的产率分别为 $0.4 \%$, $0 \%$ 和 $18.5 \%$. 如此大的产率差异可能是由于还原氧化石 墨烯具有大的比表面积 $\left(456.9 \mathrm{~m}^{2} \cdot \mathrm{g}^{-1}\right)$ 和独特的锯齿形 边缘所致, 并且这种独特锯齿形边缘很可能是催化硝基 苯还原的活性中心. 除此之外, 催化剂连续重复使用 9 次, 苯胺的产率仍高于 $95 \%$, 这说明还原氧化石墨烯对 于硝基苯还原反应是一种非常稳定高效的催化剂.

\subsection{4 -吡咯和联吡咯甲烷的合成反应}

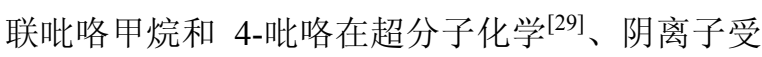
体 ${ }^{[30]}$ 和材料化学 ${ }^{[31]}$ 等领域有广泛的应用. 2011 年, Singh Chauhan 等 ${ }^{[32]}$ 直接以氧化石墨烯为催化剂, 常温条件下 首次催化合成联吡咯甲烷(Scheme 1), 研究结果表明, 其产率可高达 $80 \% \sim 99 \%$, 联吡咯甲烷在不同溶剂条件 下, 会生成不同的产物. 催化剂重复使用 3 次, 产物的 产率仅为初始催化产率的 $27 \%$.
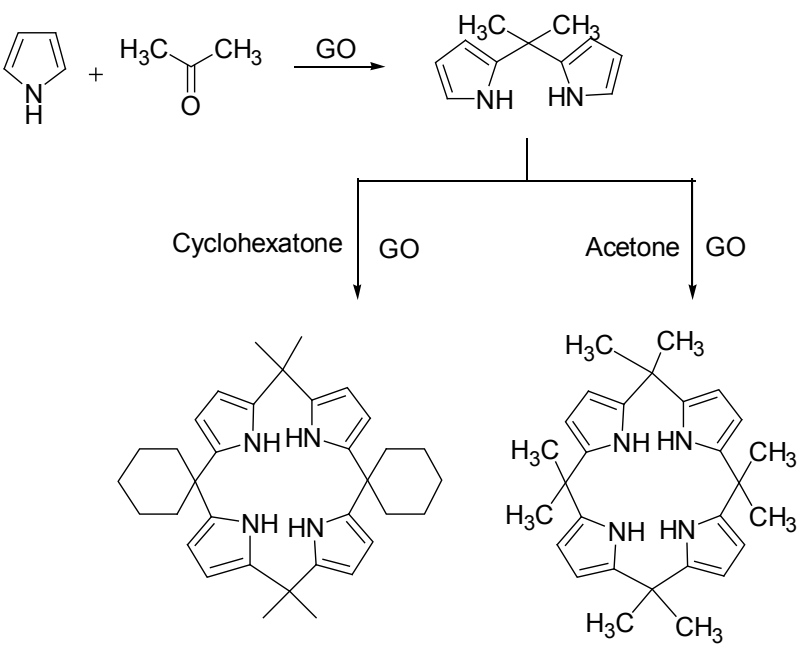

Scheme 1

\subsection{1,4-二氢吡啶的氧化反应}

吡啶类衍生物是一类具有重要生物活性的化合 
物 ${ }^{[33]} .2012$ 年, Mirza-Aghayan 等 ${ }^{[34]}$ 将氧化石墨烯用于催 化 1,4-二氢吡啶的氧化反应. 与传统的的一些催化剂: 如四吡啶合钴酸(II), 重铬酸盐, 电催化, 过二硫酸 盐- $\mathrm{Co}(\mathrm{II}), \mathrm{H}_{6} \mathrm{PMo}_{9} \mathrm{~V}_{3} \mathrm{O}_{4}, \mathrm{MnO}_{2}$ 等相比, 氧化石墨烯对连 有吸电子基和给电子基的对称(Eq. 12)或不对称(Eq. 13) 的 1,4-二氢吡啶都有极好的催化效果, 产物产率可达 90\% 96\%. 除此之外, 氧化石墨烯催化的反应时间短, 无需使用有毒的过渡金属，而且氧化石墨烯廉价易得、 成本低. 氧化石墨烯催化剂重复使用 3 次, 其催化活性 仍为初始活性的 $80 \%$ 左右.<smiles>[R]c1c(C(=O)OC)c(C)nc(C)c1C(=O)OCC(C)C</smiles>

$\mathrm{R}=\mathrm{Ph}, p-\mathrm{Me}-\mathrm{C}_{6} \mathrm{H}_{4}, p-\mathrm{Br}-\mathrm{C}_{6} \mathrm{H}_{4}, \quad 90 \% \sim 96 \%$ $m-\mathrm{OH}-\mathrm{C}_{6} \mathrm{H}_{4}, p-\mathrm{O}_{2} \mathrm{~N}-\mathrm{C}_{6} \mathrm{H}_{4}$

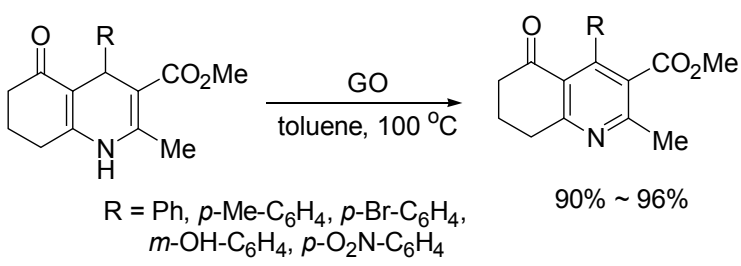

\section{4 苯甲醇脱水反应.}

2013 年, $\mathrm{Yu}$ 等 ${ }^{[35]}$ 在无溶剂条件下, 首次以氧化石墨 烯为催化剂催化苯甲醚的脱水生成二茮醚的反应. 结果 表明, 氧化石墨烯具有较好的催化活性和选择性, 产物 产率最高可达 $85.4 \%$ (Eq. 14). 考察了催化剂用量、温度 对产物产率的影响, 结果表明, $20 \mathrm{~mL}$ 苯甲醇加 $1 \mathrm{~g}$ 石墨 烯时, 二苠醚的产率最高; 当反应温度由 $70{ }^{\circ} \mathrm{C}$ 上升到 $150{ }^{\circ} \mathrm{C}$ 时，二苠醚的产率由 $5.9 \%$ 提高到 $85.4 \%$. 选择性 也由 $70{ }^{\circ} \mathrm{C}$ 时的 $75.6 \%$ 增加至 $86.6 \%$, 而再继续升温至 $170{ }^{\circ} \mathrm{C}$ 时, 二茮醚的产率和选择性几乎保持不变. 当反 应时间为 $24 \mathrm{~h}$ 时，二苠基醚收率达到最高(85.4\%), 继 续延长反应时间，二苠基醚收率则略有下降.
2

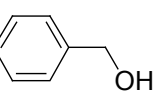

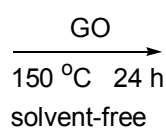

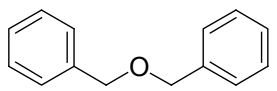

\section{3 结束语}

石墨烯及其复合材料催化的有机反应是有机化学 领域研究的一个热点, 目前其已成功应用于催化 Suzuki-Miyaura, Heck, 硝基化合物的还原以及氨硼烷 的水解脱氢等一系列重要的反应, 表现出良好的催化性 能和重复使用性能. 如何对石墨烯进行进一步的修饰, 以提高其催化活性、稳定性和重复使用性、降低催化剂
用量, 仍是今后的一个具有挑战性的课题.

\section{References}

[1] (a) Schedin, F.; Geim, A.; Morozov, S.; Hill, E.; Blake, P.; Katsnelson, M.; Novoselov, K. Nat. Mater. 2007, 6, 652.

(b) Brumfiel, G. Nature 2009, 458, 390.

(c) Sykes, E. C. H. Nat. Chem. 2009, 1, 175.

(d) Zhang, Y.-Q.; Liang, Y.-M.; Zhou, J.-X. Acta Chim. Sinica 2014, 72, 367 (in Chinese).

(张芸秋, 梁勇明, 周建新, 化学学报, 2014, 72, 367.)

(e) Lin, Y.-W.; Guo, X.-F. Acta Chim. Sinica 2014, 72, 277 (in Chinese).

(林源为, 郭雪峰, 化学学报, 2014, 72, 277.)

[2] Scheuermann, G. M.; Rumi, L.; Steurer, P.; Bannwarth, W.; Mülhaupt, R. J. Am. Chem. Soc. 2009, 131, 8262.

[3] Li, Y.; Fan, X.; Qi, J.; Ji, J.; Wang, S.; Zhang, G.; Zhang, F. Nano Res. 2010, 3, 429.

[4] Li, Y.; Fan, X.; Qi, J.; Ji, J.; Wang, S.; Zhang, G.; Zhang, F. Mater Res. Bull. 2010, 45, 1413.

[5] Siamaki, A. R.; Khder, A. E. R. S.; Abdelsayed, V.; El-Shall, M. S.; Gupton, B. F. J. Catal. 2011, 279, 1.

[6] He, Y. Q.; Zhang, N. N.; Liu, Y.; Gao, J. P.; Yi, M. C.; Gong, Q. J.; Qiu, H. X. Chin. Chem. Lett. 2012, 23, 41.

[7] Shendage, S. S.; Singh, A. S.; Nagarkar, J. M. Tetrahedron Lett. 2013, 54, 3457.

[8] Kim, S. H.; Jeong, G. H.; Choi, D.; Yoon, S.; Jeon, H. B.; Lee, S. M.; Kim, S. W. J. Colloid Interface Sci. 2013, 389, 85.

[9] Hu, J.; Wang, Y.; Han, M.; Zhou, Y.; Jiang, X.; Sun, P. Catal. Sci. Technol. 2012, 2, 2332

[10] Shendage, S. S.; Patil, U. B.; Nagarkar, J. M. Tetrahedron Lett. 2013, 54, 3457.

[11] Shang, N.-Z.; Feng, C.; Zhang, H.; Gao, S.-T.; Tang, R.; Wang, C.; Wang, Z. Catal. Commun. 2013, 40, 111.

[12] Lee, K. H.; Han, S. W.; Kwon, K. Y.; Park, J. B. J. Colloid Inerface Sci. 2013, 403, 127.

[13] Yousef, A.; Barakat, N. A.; El-Newehy, M.; Kim, H. Y. Int. J. Hydrogen Energy 2012, 37, 17715

[14] Kilic, B.; Şencanl, S.; Metin, Ö. J. Mol. Catal. A: Chem. 2012, 361 362, 104.

[15] Metin, Ö.; Kayhan, E.; Özkar, S.; Schneider, J. J. Int. J. Hydrogen Energy 2012, 37, 8161

[16] Yang, L.; Cao, N.; Du, C.; Dai, H.; Hu, K.; Luo, W.; Cheng, G. Mater. Lett. 2014, 115, 113.

[17] Cao, N.; Su, J.; Luo, W.; Cheng, G. Catal. Commun. 2014, 43, 47.

[18] Li, J.; Liu, C.-Y.; Liu, Y. J. Mater. Chem. 2012, 22, 8426.

[19] Qu, J-C.; Ren, C.-L.; Dong, Y.-L.; Chang, Y.-P.; Zhou, M.; Chen, X.-G. Chem. Eng. J. 2012, 211 212, 412.

[20] Gupta, V. K.; Atar, N.; Yola, M. L.; Ustundag, Z.; Uzun, L. Water Res. 2014, 48, 210.

[21] Nie, R.-F.; Wang, J.; Wang, L.; Qin, Y.; Chen, P.; Hou, Z. Carbon 2012, 50, 586.

[22] He, G.-Y.; Liu, W.-F.; Sun, X.-Q.; Chen, Q.; Wang, X.; Chen, H.-Q. Mater. Res. Bull. 2013, 48, 1885.

[23] Feng, C.; Zhang, H.-Y.; Shang, N.-Z.; Gao, S.-T.; Wang, C. Chin. Chem. Lett. 2013, 24, 539.

[24] Bian, J.; Wei, X.-W.; Wang, L.; Guan, Z. P. Chin. Chem. Lett. 2011, $22,57$.

[25] (a) Ge, Q.; Jenkins, S.; King, D. Chem. Phys. Lett. 2000, 327, 125.

(b) Kadossov, E.; Burghaus, U. Catal. Lett. 2010, 134, 228.

(c) Wang, H.; Liu, C.-J. Appl. Catal., B 2011, 106, 672.

[26] Li, Y.; Yu, Y.; Wang, J-G.; Song, J.; Li, Q.; Dong, M.; Liu, C.-J. 
Appl. Catal., B 2012, 125, 189.

[27] Zhang, C.; Lv, W.; Yang, Q.; Liu, Y. Appl. Surf. Sci. 2012, 258, 7795.

[28] Gao, Y.; Ma, D.; Wang, C.; Guan, J.; Bao, X. Chem. Commun. 2011, 47, 2432.

[29] Garg, B.; Bisht, T.; Chauhan, S. M. S. J. Inclusion Phenom. Mol. Recognit. Chem. 2011, 70, 249.

[30] Gale, P. A. Acc. Chem. Res. 2011, 44, 216.

[31] Gale, P. A.; Tong, C. C.; Haynes, C. J.; Adeosun, O.; Gross, D. E.;
Karnas, E.; Sedenberg, E. M.; Quesada, R.; Sessler, J. L. J. Am. Chem. Soc. 2010, 132, 3240.

[32] Chauhan, S. M. S.; Mishra, S. Molecules 2011, 16, 7256.

[33] Jia, H. P.; Dreyer, D. R.; Bielawski, C. W. Adv. Synth. Catal. 2011, 353, 528.

[34] Mirza-Aghayan, M.; Boukherroub, R.; Nemati, M.; Rahimifard, M. Tetrahedron Lett. 2012, 53, 2473.

[35] Yu, H.; Wang, X.; Zhu, Y.; Zhuang, G.; Zhong, X.; Wang, J.-G. Chem. Phys. Lett. 2013, 583, 146.

(Qin, X.) 